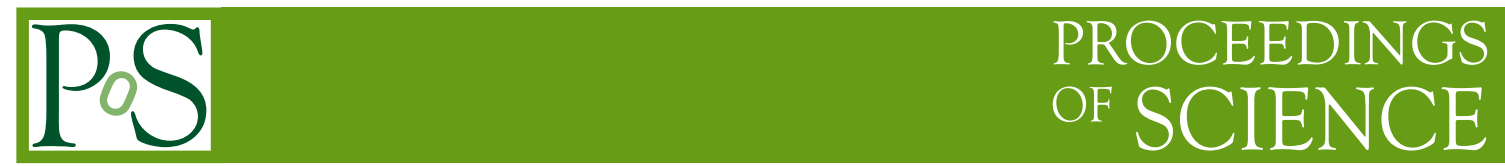

\title{
Accelerators for HEP: Challenges and R\&D
}

\author{
Vladimir Shiltsev ${ }^{a, *}$ \\ ${ }^{a}$ Fermi National Accelerator Laboratory, \\ PO Box 500, Batavia, IL 60510, USA \\ E-mail: shiltsev@fnal.gov
}

Particle accelerators are arguably the most effective tools for the particle physics research. The physics needs continuously push us to invent novel ways to increase energy and improve performance of accelerators, reduce their cost and make them more power efficient. Here we briefly overview three main families of modern and future accelerators for HEP - high intensity accelerators for neutrino research, particle factories for the electro-weak physics and Higgs studies, and post-LHC energy frontier colliders - and discuss corresponding ongoing or planned accelerator $\mathrm{R} \& \mathrm{D}$ topics and objectives.

40th International Conference on High Energy physics - ICHEP2020

July 28 - August 6, 2020

Prague, Czech Republic (virtual meeting)

\footnotetext{
${ }^{*}$ Speaker
} 
Growing demands of the high energy physics call for more powerful, more productive and more sophisticated particle accelerators. Many beam physicists and accelerator engineers carry out diverse accelerator R\&D not only to push the performance of existing machines, but also to address critical questions and issues related to the feasibility of energy, cost, performance, and power efficiency of various future accelerators. Particularly challenging are the proposed facilities that go beyond current "state-of-the-field" represented by the Large Hadron Collider with its $6.5 \mathrm{TeV}$ energy per beam, $2 \cdot 10^{34} \mathrm{~cm}^{-2} \mathrm{~s}^{-1}$ luminosity and some $1 \mathrm{TWh}$ of annual electric energy consumption. Here we overview modern and future high intensity accelerators for neutrino research, Higgs/electroweak factories and multi-TeV energy frontier colliders and outline main directions and objectives of corresponding accelerator R\&D. Our analysis mostly follows Refs.[1-3] and references many reports presented at this ICHEP2020 conference.

\section{High Intensity Accelerators for Neutrino Research}

The main types of accelerators for high energy neutrino research are superbeams and neutrino factory. Superbeams [4] are based on conventional beam dump technique: an intense high energy proton beam is directed onto a thick nuclear target producing mostly pions and kaons, which are captured by an optical system of magnetic horns in order to obtain well directed beam of same charge secondaries. High-energy neutrino beams are products of the decays of charged pions and kaons in a long decay channel. Superbeams strive to operate with a proton beam intensity closer to the mechanical stability limit of the primary targets which is at present $O(1 \mathrm{MW})$.

At neutrino factories, 20 to $50 \mathrm{GeV}$ muons generated in pion decays same way as in superbeams, are stored in a storage ring with long straight sections [5]. Decaying muons produce well-directed neutrino beams with a precisely known mixture of neutrino types.

\begin{tabular}{lccccc}
\hline Facility & $E_{b}, \mathbf{G e V}$ & $T_{c y c l e}, \mathbf{s}$ & $N_{p p p}, \mathbf{1 0}^{13}$ & $P_{b}, \mathbf{M W}$ & Year \\
FNAL MI & 120 & 1.33 & 5.2 & 0.766 & 2020 \\
(PIP-II) & 120 & 1.2 & 7.6 & 1.2 & 2027 \\
(PIP-III) & 120 & 1.2 & 15 & 2.4 & 2030 's \\
J-PARC & 30 & 2.48 & 25 & 0.515 & 2020 \\
& 30 & 1.16 & 43 & 1.3 & 2028 \\
\hline ESS $v$ SB & 2 & 0.071 & 110 & 5 & ca.2030 \\
ORKA/P & 70 & 7 & 5 & 0.09 & ca.2026 \\
& 70 & 7 & 25 & 0.45 & ca.2035 \\
ENUBET & 400 & 5.8 & 2.25 & 0.51 & (tbd) \\
\hline$\nu$ STORM & 100 & 3.6 & 4 & 0.16 & (tbd) \\
$25 \mathrm{GeV} v$-Factory & 5 & 0.02 & 10 & 4 & (tbd)
\end{tabular}

Table 1: Modern and future proton accelerators for high energy neutrino research.

At present, the leading high-energy high- power accelerators for neutrino research are rapid cycling synchrotron (RCS) facilities J-PARC in Japan which has reached $0.515 \mathrm{MW}$ of the $30 \mathrm{GeV}$ proton beam power [6], and the Fermilab Main Injector delivering up to $0.766 \mathrm{MW}$ of $120 \mathrm{GeV}$ 
protons [7]. There are several promising new proposals under consideration including ESSvSB, Protvino/ORKA, ENUBET, and the $v$ STORM - see Table 1. The latter will represent the first step towards future neutrino factories and is also synergistic with world-wide muon collider accelerator physics R\&D activities (see below).

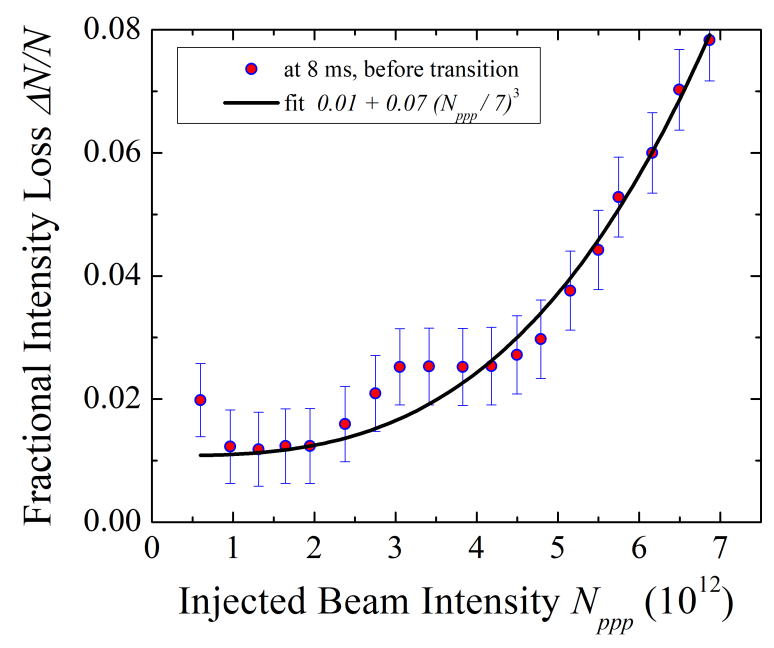

Figure 1: Intensity-dependent fractional FNAL Booster $400 \mathrm{MeV}$ proton beam intensity loss shortly after injection vs total number of protons (from [8]).

The needs of neutrino physics call for the next generation, higher-power, megawatt and multiMW-class superbeams facilities. Average proton beam power on the neutrino target scales with the beam energy $E_{b}$, number of particle per pulse (PPP) $N_{p p p}$ and cycle time $T_{c y c l e}$ as $P_{b}=$ $\left(E_{b} N_{\text {ppp }}\right) / T_{\text {cycle }}$. One way to increase $P_{b}$ is to increase the beam energy. That would require either better or new magnets and RF acceleration system, and/or to decrease the cycle time (again, new magnets and RF might be needed) - that usually implies high cost and significant increase of the facility AC power consumption. There are several approaches actively pursed by the RCS machine designers and engineers for the power upgrades and future machines such as more efficient SC magnets, like $4 \mathrm{~T} / \mathrm{s}$ ones for the FAIR project in Darmstadt (Germany) built with NbTi SC cables [9] or 12 T/s HTS-based magnet prototype recently tested at FNAL [10]; FFAG (fixed field alternating gradient) accelerators [11]; also, under development are more efficient power supplies with capacitive energy storage and recovery, and on more economical RF power sources such as $80 \%$ efficient klystrons, magnetrons, and solid-state ones (compare to current $\sim 55 \%$ ) [12].

The beam power can also be boosted by increasing the PPP - then, the key challenges will be mostly associated with numerous beam dynamics issues and, sometimes, with the cost. The utmost PPP challenge is about how to control the beam losses below $W \sim 1 \mathrm{~W} / \mathrm{m}$ while increasing intensity $N_{\text {ppp }}$. That requires the fractional beam loss scale down with increase of beam intensity, energy and power $\left(\Delta N_{p p p} / N_{p p p}\right) \leq W / P_{b} \sim W /\left(N_{p p p} \gamma\right)$. In reality, the repelling forces of the proton beam's own space-charge lead to increase of beam sizes and particle losses at higher beam intensities - see Fig.1. Accelerator R\&D explores several lines of attack on the problem: i) to increase the injection energy into the RCS - that allows to gain the intensity $N_{p} \sim \beta \gamma^{2}$, but requires (often $\mathrm{SRF}$ ) injection linacs; ii) to employ larger aperture magnets which would allow certain blowup of 
the transverse rms size $\sigma$ without the beam halo hitting the aperture and resulting in the losses that might be expensive; iii) to flatten the beam current pulse shape by using the 2 nd harmonics $\mathrm{RF}$ and reduce $B_{f}$; iv) to use the transverse painting (shaping) of the beams via charge-exchange injection to linearize the space-charge forces; v) to design better efficiency collimation system to lower uncontrolled irradiation (due to the particles which might avoid dedicated collimators or dumps and scattered around the ring); vi) to make the beam focusing lattice perfectly periodic, e.g., like in the Fermilab Booster RCS with periodicity of $P=24$, or in the J-PARC MR with $P=3$; vii) to introduce Non-Linear Integrable Optics elements to reduce the losses [13]; viii) to employ electron lenses for the space-charge compensation [14]. The last two approaches are novel and are being tested at the dedicated IOTA ring at Fermilab [15]. There are other intensity dependent issues in the RCSs for HEP, such as efficient injection of high power beams (that requires stripping electrons off $H^{-}$particles by foils or lasers), electron cloud effects and coherent beam instabilities.

In all scenarios, one would need reliable targets and horns to shape the secondary beams. There the challenge is that due to the radiation damage and thermal shock-waves, the operational lifetime of the targets gets worse (shorter) with the power increase and frequent replacements of them may pose serious impediment on facility's uptime. Existing neutrino targets and horns are good to about $0.8 \mathrm{MW}$ beam power, while MW and multi-MW targets are under active development and prototyping. Ongoing R\&D program includes studies of material properties, new forms (foams, fibers), new target designs (e.g., rotating or liquid targets) [16].

The neutrino factory (NF) concept envisions very high intensity neutrino and antineutrino beams which are exact CP conjugates - a total of $O\left(10^{21}\right)$ muon decays per year [17]. The NF performance depends on the power of the proton driver and the degree or absence of the muon cooling. The ionization cooling method though relatively straightforward in principle, has some practical implementation challenges such as RF breakdown suppression and attainment of high accelerating gradients in relatively low frequency normal-conducting RF cavities immersed in strong magnetic fields. While key NF beam physics challenges have been addressed, several important accelerator technology and cost feasibility issues are subject of ongoing $R \& D$ and conceptual design optimization $[18,19]$.

The basic idea for $v$ STORM facility is production of neutrino beams from the decay of $1 \mathrm{GeV} / \mathrm{c}$ to $6 \mathrm{GeV} / \mathrm{c}$ muons in a racetrack-like decay ring, i.e., like in a neutrino factory but without muon cooling and acceleration [20,21]. The major challenges of the proposal are a) necessity to have a large diameter $(0.5 \mathrm{~m})$ magnets to accept most of the secondary muons, and b) a sophisticated focusing lattice which should assure survival of about $60 \%$ of muons after 100 turns with the $10 \%$ rms beam momentum spread. Conceptually close to $v$ STORM is the idea of Beta-beams which exploits the $\beta$-decay of relatively short-lived radio-isotopes such as ${ }^{18} \mathrm{Ne}$ or ${ }^{6} \mathrm{He}$ with half-lives of around $1 \mathrm{~s}$ which are ionised, accelerated to relativistic speeds and put into a racetrack storage ring, where they eventually decay [17].

\section{Colliders for Electro-weak and Higgs Physics}

Big number of the future colliders proposals has been brought up in the course the 2020 European Particle Physics Strategy Update discussions [3] and the 2021 Snowmass US HEP planning process [22]. Fig.2 presents proposed timelines of some of them. Linear collider Higgs/EW fac- 
tories, such as $250 \mathrm{GeV}$ c.m.e. ILC [23] and CLIC [24] are among the most advanced projects, being at the readiness level of the Technical Design Report (TDR) readiness and the Conceptual Design Report (CDR), respectively. All their major R\&D have been completed and they are in the "ready-for-approval" and pre-construction project stage. Besides large scale operation of thousands the SC or NC RF units, their major concerns are mostly about their luminosity, which is strongly dependent on efficient positron production (exceeding by more than an order of magnitude the level achieved at the SLC - see Fig.3) and the anticipated commissioning time needed to learn how to effectively operate these machines, which are very sensitive to ground motion and other noises. Note also, the the CLIC two-beam acceleration scheme is novel and the project has klystrons-based backup option. Circular $e^{+} e^{-}$Higgs factories, such as FCCee and CEPC [25] have satisfactorily

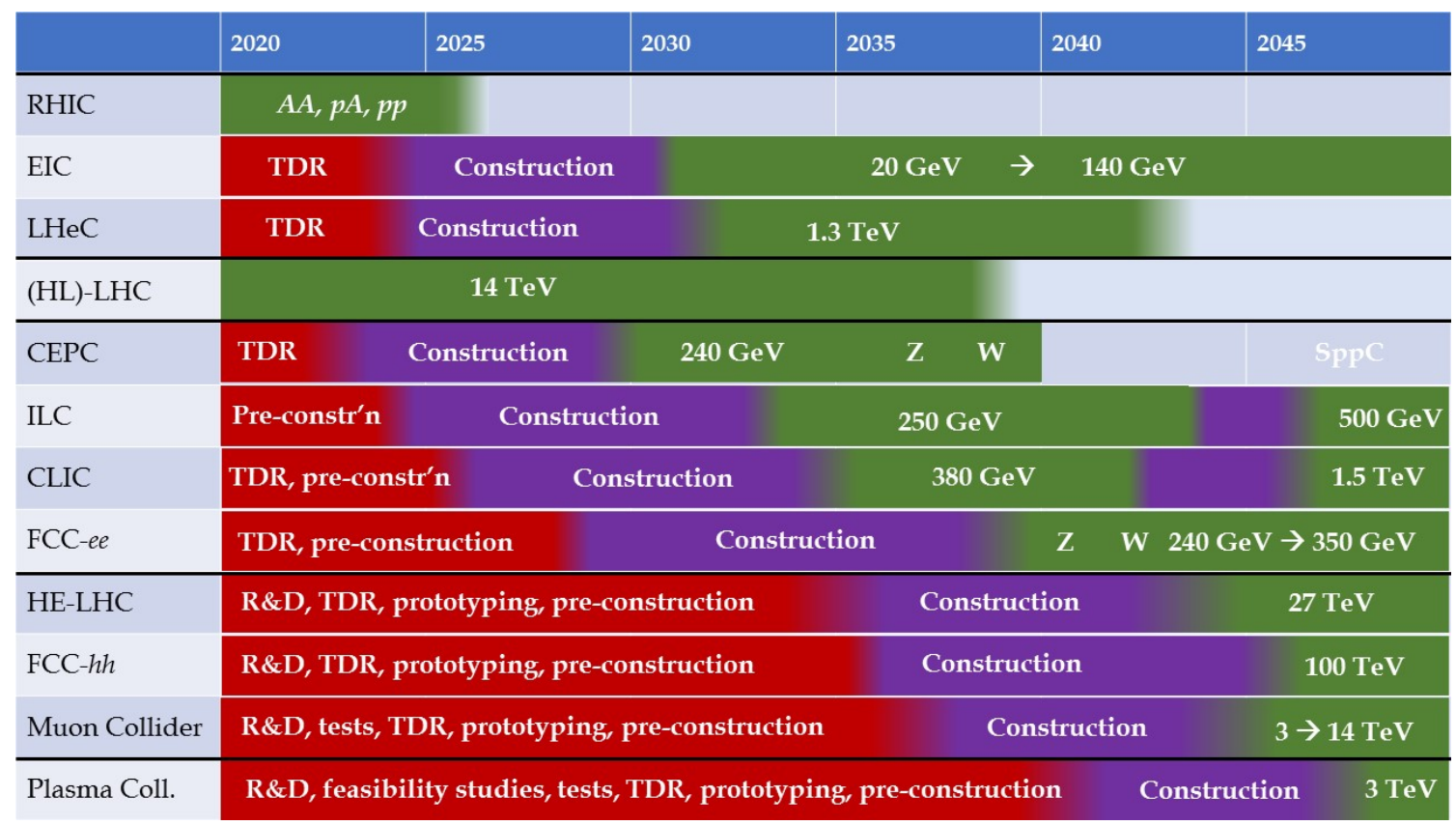

Figure 2: Plans of operational high energy particle colliders and approximate technically limited timelines of future large colliding beam facilities (from [2]).

addressed general feasibility issues, they have accomplished CDRs and now enter the multi-year stage of developments toward TDRs to prototype major critical systems and to address in detail the matters of financial feasibility, realistic timelines and expected performance. Major challenges of these machines are related to the cost, driven by the SRF systems, magnets, and civil construction; as well as the energy efficiency as at present these projects foresee the need of about $300 \mathrm{MW}$ of the site electric power. Strong international R\&D collaborations are in place, e.g., the FCC team, and their main R\&D priorities include [26]: 1) development of $80 \%$ efficient klystrons and high efficiency CW SC cavities; 2) energy recycling/storage systems for high efficiency rapid cycling booster magnets; 3) non-linear focusing lattice and beam dynamics analysis with beam-beam effects, beamstrahlung and imperfections and the dynamic aperture optimization with the nanobeams IP scheme. Common for these linear and circular colliders is the energy efficiency concern, e.g., in the terms of luminosty per TWh of the wall-plug electric energy consumed (see Fig.4). 


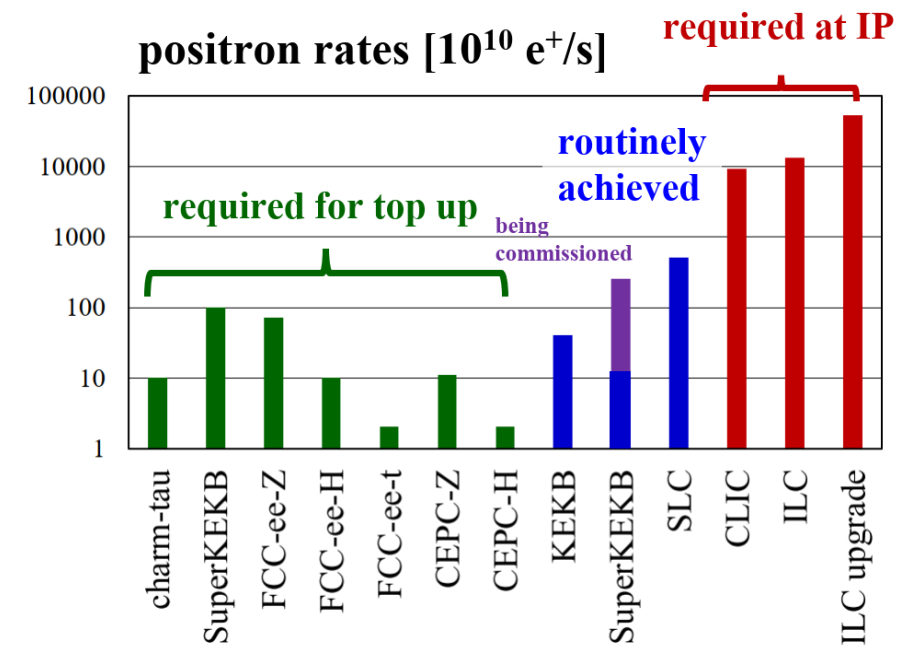

Figure 3: Positron production rates achieved at the SLC, KEKB and SuperKEKB compared with the need for top-up injection at future circular and linear $e^{+} e^{-}$colliders (from [2]).

\section{Energy Frontier Multi-Tev Colliders}

The most critical requirement for the post-LHC energy frontier colliders is the center-of-mass energy reach and there are five leading proposals (in order of the energy reach): $3 \mathrm{TeV}$ CLIC, 27 TeV HE-LHC [27], 3 to 14 TeV Muon Colliders [28], [29], [30], 75 TeV SppC and 100 TeV FCC-hh. The construction cost is the lowest for the HE-LHC and the Muon Collider, followed by CLIC-3 and FCC-hh, all within a factor of 2 to 3 [2]. The required AC site power consumption is the lowest for the HE-LHC and the Muon Collider, followed by ILC, then by FCC-hh and CLIC, all within a factor of 2 to 3 . As for the required duration and scale of the R\&D effort to reach the TDR level of readiness, the CLIC-3 project would need some 10 years of $\mathrm{R} \& \mathrm{D}$, while about twice that time is required for the HE-LHC, FCC-hh/SppC, and for the Muon Collider.

Development of a representing magnet with up to $16 \mathrm{~T}$ maximum field is arguably the hardest challenge for the hadron colliders in view of fundamental challenges in getting the required current density in superconductors and in dealing with the ultimate magnetic pressures and mechanical stresses in the superconductor and associated components. It is estimated that development of $\mathrm{Nb} 3 \mathrm{Sn}, 14 \mathrm{~T}$ to $16 \mathrm{~T}$ magnets will require $10-15$ years for short-model $\mathrm{R} \& \mathrm{D}$, followed by 10 to 15 years of prototyping/pre-series with industry [3]. The current world record of 14.5 Teslas for field strength for a superconducting accelerator dipole magnet was achieved by Fermilab team in 2020. The SppC team plans to explore feasibility of an alternative low-cost $12 \mathrm{~T}$ dipoles built with an iron-based superconductor. Other challenges, demanding substantial R\&D efforts include: a) efficient intercept of the synchrotron radiation power (5 MW in FCC-hh / 1 MW SppC); b) precise and safe collimation of the ultra-high energy beams with some 7 times the LHC circulating beam power; c) non-linear lattice studies to increase dynamic aperture at injection (either 1.3 TeV SC-SPS or a new 3.3 TeV SC injector); d) optimal IR design and MDI (machine-detector interface) studies, etc.

Muon colliders with cme up to $14 \mathrm{TeV}$ - see Fig. 5 - offer about factor of 7 advantage in 


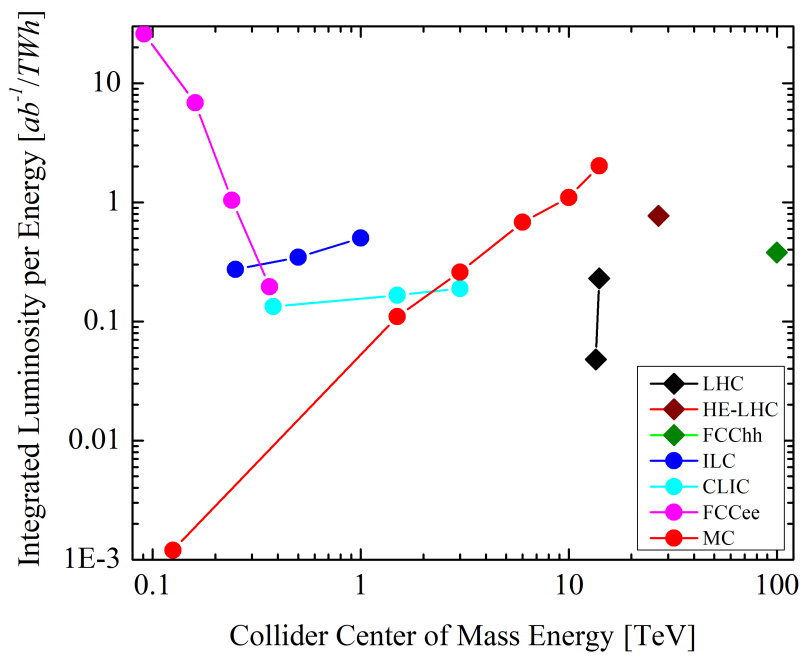

Figure 4: Energy efficiency of present and future colliders. Annual integrated luminosity per Terawatt-hour of electric power consumption as a function of the centre-of-mass energy. The LHC - both present and expected after its high-luminosity upgrade (black diamonds) - is contrasted with a variety of proposed particle colliders, as taken from reference [2]: the Muon Collider (MC, red circles), the Future Circular electron--positron Collider (FCC-ee, magenta circles) assuming experiments at two collision points, the International Linear Collider (ILC, blue circles), the Compact Linear Collider (CLIC, cyan circles), the High Energy LHC (HE-LHC, magenta diamonds), and the Future Circular proton-proton Collider (FCC-hh, green diamonds). The effective energy reach of hadron colliders (LHC, HE-LHC and FCC-hh) is approximately a factor of seven lower than that of a lepton collider operating at the same energy per beam (from [31]).

the potential mass reach for the exploration of the energy frontier, in addition to being attractive because of better $\mathrm{ab}^{-1} / \mathrm{TWh}$ efficiency, smaller size and cost [31]. However, muons are produced as secondary particles and decay rapidly, so they have to be collected, cooled and accelerated rapidly that presents a special challenge for a collider. Recent research indicates that the technologies required to overcome this challenge are within our grasp. The MERIT experiment at CERN has demonstrated that the liquid Mercury target system can sustain an intense primary proton beam of 4MW power and more [32]. The International Muon Ionization Cooling Experiment (MICE) at RAL (UK) has recently demonstrated effective $O(10 \%)$ reduction of transverse emittance of initially dispersed $140 \mathrm{MeV} / \mathrm{c}$ muons passing through an ionization cooling channel cell consisting of a sequence of $\mathrm{LiH}$ or liquid Hydrogen absorbers within a lattice of up to $3.5 \mathrm{~T}$ solenoids that provide the required particle focusing [33]. Further R\&D is required on: i) effective muon production and 6D cooling; ii) fast acceleration (magnets, RF), iii) detector background reduction and machine detector interface, iv) large aperture $12 \mathrm{~T} \mathrm{SC}$ magnets, v) countermeasures to minimize the radiation effects of neutrinos, produced by decaying muons.

A CERN-led international collaboration is being formed to bring together the diverse expertise and complementary capabilities from around the world to realize the muon collider as the nextgeneration energy-frontier discovery machine. The objectives of the collaboration are i) to develop, in about 4 years, the CDR of a baseline $3 \mathrm{TeV}$ collider with a luminosity of $2 \cdot 10^{34} \mathrm{~cm}^{-2} \mathrm{~s}^{-1}$ and 


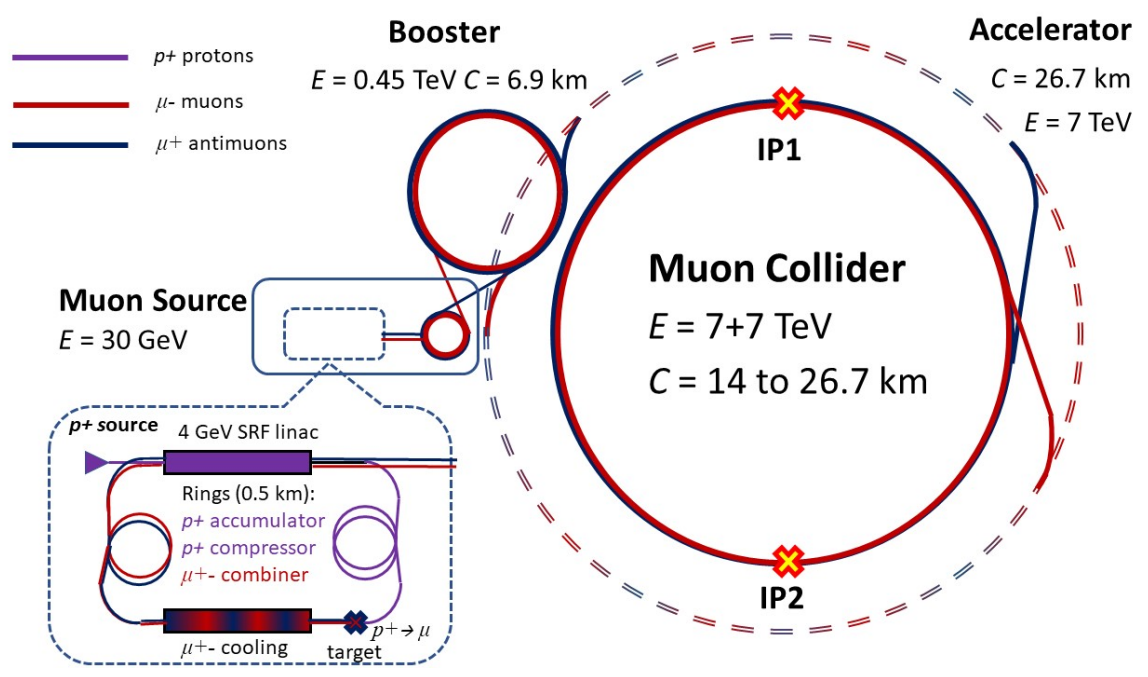

Figure 5: Schematic layout of a $14 \mathrm{TeV}$ muon collider complex. The muon injector systems include the proton driver, a high-power target system with a capture solenoid for the pions generated by the proton interactions with the target, a pion decay channel, where muons are collected and subsequently bunched together, a muon ionization cooling channel that provides cooling for both positive and negative muon beams by more than five orders of magnitude, a low energy muon accelerator stage that would deliver beams with energies up to $30 \mathrm{GeV}$, followed by a $450 \mathrm{GeV}$ high energy injector. From the injector, each species of muon beam is transferred into a high-energy accelerator complex that can increase the beam energy to the multi-TeV range and can possibly share the $26.7 \mathrm{~km}$ tunnel with the muon collider ring. Finally, the beams will be transferred to the collider ring, whose circumference, optimized for cost considerations and luminosity performance, can be from $26.7 \mathrm{~km}$ to about $14 \mathrm{~km}$ [31]. A $14 \mathrm{TeV}$-class collider ring is anticipated to support at least two detector interaction regions for the particle physics program.

a 10-14 TeV collider with a luminosity of $4 \cdot 10^{35} \mathrm{~cm}^{-2} s^{-1}$ (the discovery potential of the $>10 \mathrm{TeV}$ machine would be competitive to or exceed that of any other energy frontier collider option being evaluated at the moment), ii) to construct a comprehensive facility for the most relevant tests and studies, and iii) to come up with a Technical Design Report by the end of 2030's.

Many innovative accelerator concepts are now under active exploratory studies. Among them are: i) Energy Recovery Linac (ERL) based FCCee [34] assumes $100 \mathrm{~km}$ long $240 \mathrm{GeV} \mathrm{cme} e^{+} e^{-}$ collider, that brings particles' energies up in just several turns employing $60 \mathrm{GeV}$ per turn SRF accelerating sections and promises some 3-10 times less RF power per $\mathrm{ab}^{-1}$ of luminosity than the FCCee; main challenges of such approach are the need for generation and control of very small beam emittances as well as strong beam-beam effects; ii) ERL-based LHeC (or FCCeh) [35] also seeks acceleration of electrons in three turns to $30-60 \mathrm{GeV}$ by SC RF sections in a new $6 \mathrm{~km}$ tunnel to collide with the LHC/FCCpp protons and deliver $O\left(1 \mathrm{ab}^{-1}\right)$ of luminosity at 1.2/3.2 TeV c.m.e.; extensive tests of the ERL technology are planned at the PERLE facility at Orsay; iii) $\gamma-\gamma$ Higgs Factories [36] require collisions of only $80 \mathrm{GeV} e^{-}$for the Higgs production, but require further $\mathrm{R} \& \mathrm{D}$ on the design of such recirculating linear accelerator facilities, on the high power lasers, etc; iv) in the Gamma-Factory concept [37] collision of high intensity laser with the LHC ions results in generation of $O(1 \mathrm{GeV}) \gamma$ 's, positrons and muons; following recent proof-of-principle demonstration, possibilities of significantly higher fluxes are being considered. 
Particle acceleration by plasma waves has enormous promise for unmatched gradients and may turn into the most advanced practical technology. Impressive proof-of-principle experiments of the past two decades have demonstrated three effective ways to excite plasma: i) by short laser pulses with energy gains up to $4.3 \mathrm{GeV}$ over $9 \mathrm{~cm}$ in $10^{18} \mathrm{~cm}^{-3}$ plasma; ii) by intense electron bunches $\left(9 \mathrm{GeV}\right.$ over $1.3 \mathrm{~m}$ at $\left.10^{17} \mathrm{~cm}^{-3}\right)$; and iii) by high energy proton bunches $(2 \mathrm{GeV}$ over $10 \mathrm{~m}$ at $10^{15} \mathrm{~cm}^{-3}$ ). In principle, plasma-based linear accelerators can be employed for high energy $e^{+} e^{-}$ colliders, but significant long-term R\&D effort is required to address many critical issues, such as acceleration of positrons, beamstrahlung, staging efficiency, power efficiency, emittance control vs vibrations, jitter and scattering in plasma, etc. There are several very active collaborations worldwide which are focused on these issues as well on possible early non-collider applications of the plasma accelerators: ALEGRO, BELLA, FACET-II, EuPRAXIA, and others [38], [39], [40].

\section{Acknowledgements}

I greatly appreciate my long-time collaboration with F.Zimmermann of CERN on the entire spectrum of issues related to modern and future colliders, that resulted in the recent review article widely cited here. Very helpful were also discussions with S.Gourlay (LBNL), T.Raubenheimer (SLAC), D.Denisov (BNL) and M.Narain (Brown University) within the US Snowmass'21 planning process.

Fermi National Accelerator Laboratory is operated by the Fermi Research Alliance, LLC under Contract No. DE-AC02-07CH11359 with the United States Department of Energy.

\section{References}

[1] V.Shiltsev, Modern Physics Letters A, 35 (17), 2030005 (2020).

[2] V.Shiltsev, F.Zimmermann, arxiv:2003.0908 (to appear in Reviews of Modern Physics, 2020)

[3] R.K.Ellis, et al., arxiv:1910.11775; The European Strategy Group, CERN-ESU-013 (2020).

[4] S. Kopp, Phys. Reports 439 (3), 101 (2007).

[5] S. Geer, Phys. Rev. D 57(11), 6989 (1998).

[6] S.Sekiguchi, et al., this Conference, contribution ID 775 (2020).

[7] T.Jones, this Conference, contribution ID 913 (2020).

[8] V.Shiltsev, J.Eldred, V.Lebedev, K.Seiya, Preprint FERMILAB-TM-2740 (2020).

[9] E. Fischer, et al., Proc. IPAC 2012 (New Orleans, USA), pp.3535-3537 (2012)

[10] H. Piekarz, et al., NIM A 943, 162490 (2019).

[11] Y.Mori, NIM. A, 562 (2), 591-595 (2006).

[12] V. Yakovlev, et al., Proc. IPAC 2017 (Copenhagen, Denmark), pp.4842-4827 (2017) 
[13] V. Danilov, S. Nagaitsev, Phys. Rev. S.T.-A.B. 13 (8), 084002 (2010).

[14] V. Shiltsev, Electron lenses for super-colliders (Springer, 2015).

[15] S.Antipov, et al., JINST 12, T03002 (2017).

[16] R. Zwaska, et al., Proc. IPAC 2018 (Vancouver, Canada), MOZGBE2 (2018)

[17] T. Edgecock, et al., Phys. Rev. S. T. Accel. Beams, 16(2), 021002 (2013).

[18] S. Geer, Ann. Rev. Nucl. Part. Sci. 59, 347 (2009).

[19] J.P. Delahaye, et al., Journ. Instr. 13(06), T06003 (2018).

[20] D. Adey, R. Bayes, A. Bross, P. Snopok, Ann. Rev. Nucl. Part. Sci. 65, 145 (2015).

[21] K. Long, et al., EPPSU Input 154, https://indico.cern.ch/event/765096/contributions/

[22] https://www.snowmass21.org/accelerator/start

[23] G.Eigen, this Conference, contribution ID 346 (2020).

[24] P.Burrows, this Conference, contribution ID 782 (2020).

[25] H.Shi, et al., this Conference, contribution ID 338 (2020).

[26] J.Gao, this Conference, contribution ID 615 (2020).

[27] L.Malina, et al., this Conference, contribution ID 601 (2020).

[28] J.Pasternak, this Conference, contribution ID 53 (2020).

[29] N.Pastrone, et al., this Conference, contribution ID 640 (2020).

[30] F.Collamati, this Conference, contribution ID 795 (2020).

[31] K.Long, et al., arxiv:2007.15684 (submitted to Nature Physics, 2020).

[32] K. McDonald, et al., Preprint FERMILAB-CONF-10-212-APC (2009).

[33] M. Bogomilov, et al., Nature 578(7793), 53 (2020).

[34] V.Litvinenko, T.Roser, M.Chamizo-Llatas, Physics Letters B, 804, 13594 (2020).

[35] B.Holzer, this Conference, contribution ID 729 (2020).

[36] SA Bogacz, et al., arXiv:1208.2827

[37] M.Krasny, this Conference, contribution ID 7771 (2020).

[38] A.Molodozhentsev, et al.,, this Conference, contribution ID 1001 (2020).

[39] M.Wing, et al.,, this Conference, contribution ID 105 (2020).

[40] F.Schilacci, et al., , this Conference, contribution ID 1037 (2020). 